\title{
Safeguards and Pitfalls in Technique Used for Stem Cell Delivery in Children Suffering from Idiopathic Dilated Cardiomyopathy
}

\author{
Aris Lacis ${ }^{1}$, Inguna Lubaua ${ }^{2}$, Inta Bergmane ${ }^{1}$, Eriks Jekabsons ${ }^{3}$, Janis Ancans ${ }^{4}$, Andis Lacis ${ }^{2}$ and Andrejs Erglis ${ }^{5}$ \\ 1. Clinic of Pediatric Cardiology and Cardiac Surgery, University Hospital for Children, Riga 1004, LV, Latvia \\ 2. Riga Stradins University, Riga 1007, LV, Latvia \\ 3. Pauls Stradins Clinical University Hospital, Riga 1002, LV, Latvia \\ 4. University of Latvia, Riga 1586, LV, Latvia \\ 5. The Latvian Institute of Cardiology, Riga 1002, LV, Latvia
}

\begin{abstract}
We report 7 pediatric patients with idiopathic dilated cardiomyopathy who underwent autologous bone marrow derived mononuclear cells intramyocardial delivery as a final option after unsuccessful routine conservative treatment and have real perspective for cardiac transplantation in childhood in our country at new time. For two patients intramyocardial delivery was performed repeatedly 22 and 24 months respectively after first repair. All patients received massive anti-congestive therapy by diuretics, ACE inhibitors, carvedilol, digitalis and aspirin.
\end{abstract}

Key words: Idiopathic dilated cardiomyopathy in children, stem cell delivery methods.

\section{Introduction}

DCM (dilated cardiomyopathy) is a serious problem in pediatric cardiology. Despite the relatively low incidence $0.57 / 100,000$ to $2.6 / 100,000$ children, the mortality is high, one third of patients die within a first year after diagnosis $[1,2]$. Up to $40 \%$ of them are defined to be idiopathic dilated cardiomyopathy (IDCM) - defined as left ventricular dilatation and systolic dysfunction of unknown cause. The prognosis is poor in pediatric patients and survival appears to be related to the degree of systolic dysfunction. Medical therapy does not provide a better prognosis. Cardiac transplantation seems to be the end-stage therapy for these patients. However, the lack of donors is an important limitation of this procedure. Furthermore, these patients do not live long enough to undergo heart transplantation because of the delayed diagnosis.

Corresponding author: Aris Lācis, Ph.D., Prof., research fields: pediatric cardiology, cardiac surgery, and application of stem cell therapy. E-mail: aris.lacis@ apollo.lv.
Recent clinical studies [3, 4] suggested that bone marrow-derived autologous mononuclear cells or circulating progenitor cells $[5,6]$ may be a promising therapy for treating these patients.

Since the first human trial published in 2001 by Menasche et al. [7], a lot of trials have been reported, but till the year of 2012 only four reports with a limited amount of patients were published [3-6]. The mentioned trials experimented with different routes of delivery, including direct intramyocardial injection at the time of coronary artery bypass grafting [8,9], intra-coronary infusion [3-6] and percutaneous endocardial injection using specific devices such as the NOGA catheter [10-12].

\section{Materials and Methods}

We have started intramyocardial stem cell transplantation in collaboration with The Latvian Center of Cardiology, Pauls Stradins Clinical University Hospital and Cell Transplantation Center 
since May 2009. The agreement of ethics committee was received. Cell suspension preparation: manufacturing is performed at The Latvian Laboratory of Cell Transplantation of Pauls Stradins Clinical University Hospital. The manufacturing procedures are in compliance with principles of good manufacturing practices as set out in Directive 2003/94/EC (3-year experience in processing biological medical products). A manufacturing authorization, Certificate Nr. AO-3, registration 2453/3817, including manufacturing of bone marrow derived cell therapy products. There were 17 patients with dilated cardiomyopathy in our center since 2000. Six of them are died, one underwent the Batista procedure in another center and three recovered. We performed cell therapy for all the patients who received the medical therapy of heart failure and newly diagnosed cases. The decision to perform bone marrow progenitor cell intramyocardial implantation was made due to no perspective condition of the patient and no possibilities for heart transplantation. Following detailed, multiple observations of the first patient over one year, the decision to use the method in other patients was made. Informed consent from the parents was received. Already seven patients ranging in age from 4 months to 17 years and weight from 5 $\mathrm{kg}$ to $61 \mathrm{~kg}$ had been admitted for the transplantation of bone marrow derived progenitor cells, three of them at age of 4 months. We chose patients with severe and moderate decrease in ejection fraction. The decreased heart function was established by $2 \mathrm{D}$ and 3D echocardiography: LVDd more than 2SD in Z-score, decreased FS $(<27 \%)$ and EF $(<45 \%)$. The inflammatory and autoimmune processes were excluded by laboratory investigations (WBC, CRO, ANA at normal range), also genetically determined metabolic disorders were excluded, and the virus (RSV, adeno, parvo B19, entero, CMV, EBV, rubella, rubeola) antibodies were negative for all of them. Transvenous endomyocardial biopsy via femoral vein was done for 2 patients. The histological investigation showed a myocytes hypertrophy and fibrosis. All patients received massive anti-congestive therapy by diuretics, ACE inhibitors, carvedilol, digitalis and aspirin.

\subsection{Preparation of Bone Marrow Aspirate}

5 to $30 \mathrm{mLs}$ of bone marrow was aspirated from iliac crest, 17 to 122 million BMCs were isolated. Samples for flow cytometry were taken from $15 \mathrm{~mL}$ of mononuclear cell fraction prepared for transplantation. Preparation of the isolated cells was carried out using Stem-kit ${ }^{\mathrm{TM}}$ reagents (Cat. Nr. IM3630; Beckman Coulter), that contained CD34-PE, CD45-FITCm isotype control, 7AAD (viability dye) and Stem-Count Fluorospheres. FACS analysis was performed on FC-500 (Beckman Coulter) and analyzed using CXP analysis software. Each measurement contained at least 50,000 events. Maximum number of events was 100,000. Obtained numbers of cells $/ \mu \mathrm{L}$ were calculated for total number of $\mathrm{CD} 34+$ cells within transplantation material. Measurements with less than 50,000 events were excluded from study.

\subsection{Method for Delivery}

The material as suspension of physiologic saline was given to patients by intramyocardial puncture in two doses, each $1 \mathrm{~mL}$. The decision to divide volume in two doses was made to minimize the barotrauma of tissue. We did not want make injection in free wall due to risk of perforation. The transcutan injection was performed in general anesthesia from subxiphoid position through anterior wall and right ventricle cavity in myocardium of interventricular septum or region of apex under echocardiographic control at the operating room. The $0.95 \times 220 \mathrm{~mm}$ CHIBA needle was used for injection. There were no observed any complications of procedure.

\subsection{Postdelivery Examination}

Every patient underwent repeated examination 
every two months, including physical examination, electrocardiography (ECG), and transthoracic echocardiography. The data of six patients had a complete one year follow-up are analyzed in present study. The statistical significance was determined by $t$-Test $(P<0.05)$.

\section{Results}

During one year following BNCs transplantation, we observed increase of ejection fraction. The average basal EF was $33.5 \%$. We observed increasing up to $54 \%(P=0.00154)$ in 6-month period and up to $54.5 \%(P=0.00315)$ after one year. The difference between basic data and results after 6 and 12 months was statistically significant, but there was no difference between 6-month and 12-month data. The results were within the confidence interval in all measurements. Also there was correlation observed in ECG and echo improvement and CTR decrease. The electrocardiographic left ventricular overload was decreased. The CTR in chest X-ray decreased by $11 \%$ (from average 63.16 to $55.5 ; P=0.016$ ). The clinical status of patients improved from class III-IV to I-II (NYHA). The better effect was observed in the patient with the worse initial status and lowest ejection fraction. Despite the improvement in EF, not all patients were observed reduction of LVDd. There were no observed side effects or heart rhythm disorders.

\section{Discussion}

The current gold standard for treatment of dilated cardiomyopathy is the heart transplantation which has good results in perspective [13, 14]. Unfortunately, the transplantation is limited due to the absence of suitable donors. Of course, the ventricular assisting device is the way of bridging to transplantation. Both methods are expensive procedures and need lots of resources $[15,16]$. Other surgical options are resynchronization therapy and the Batista procedure. The resynchronization is a promising method and is considered as an alternative to delay the heart transplantation, as for the Batista procedure, the European Society of Cardiology guidelines do not recommend it for treatment of dilated cardiomyopathy [17]. Talking about endomyocardial biopsies, the views are ambiguous. The analysis of some literature data shows that endomyocardial biopsy is not suitable for diagnosis, therapy, detection of early stages or prognostic evaluation in the dilated cardiomyopathy however another sources say that endomyocardial biopsy in children remains important in the evaluation of cardiomyopathy $[18,19]$. We believe that the need for biopsy should be carefully evaluated due to high risk of myocardial perforation in infants. We were trying to find another way to improve the patients' situation without the possibility of LVAd and, at final, transplantation. Due to absence of wide clinical studies of the stem cell application in treatment of dilated cardiomyopathy, we can compare results only with few case reports. In our study we observed the increase of cardiac function like the Justus Liebig University (Giessen, Germany), Gazi University (Ankara, Turkey) and Teheran University's (Tehran, Iran) case results $[3,5,11]$. The better results were observed in younger patients and, through manipulation in the earlier period since the onset of the disease unlike those who had previously received prolonged conservative therapy only. We also noted that the vast improvement in cardiac function was observed in those patients who initially had worse data $[20,21]$. That provides the basis for future research to clarify the optimal timing and possibly the age of stem cell transplantation. The question of stem cell action mechanisms is left open: direct cell implantation or paracrine effect via cytokine induced growing and increase of residual viable myocytes, stimulation of resident cardiac stem cells or induction of cell fusion between transplanted bone marrow cells and resident myocytes. There are reports of stem cells direct replacing in myocardium as well as evidence of paracrine effect $[22,23]$. 


\section{Conclusions}

Almost half of the children with symptomatic dilated cardiomyopathy receive a transplant or die within two years. However, cardiac stem cell transplantation has become a promising therapeutic option.

The early results indicate favorable benefit-risk ratio and mortality reduction for all children with dilated cardiomyopathy after autologous bone marrow mononuclear cell therapy. Although multi-centre randomized trials remain to be considered. It can be concluded that survival benefits continue to outweigh the risks related to cardiac stem cell transplantation, if performed by experienced cardiac surgeons in appropriate hospital setting and optimal selection of time, cell types and routes of delivery.

In general, the proposed method of treatment poses a low risk and offers significant benefit for critical patients when other therapeutic options are limited or not available at all.

\section{References}

[1] J. Alvarez, J.D. Wilkinson, S.E. Lipshultz, Outcome predictors for pediatric dilated cardiomyopathy: A systemic review, Prog. Pediatr. Cardiol. 23 (1) (2007) 25-32.

[2] A. Arola, J. Tuominen, O. Ruuskanen, E. Jokinen, Idiopathic dilated cardiomyopathy in children: Prognostic indicators and outcome, Pediatrics 101 (31) (1998) 369-376.

[3] S. Rupp, J. Bauer, T. Tonn, V. Schachinger, Z. Dimmeler, A.M. Zeiher, et al., Intracoronary administration of autologous bone marrow-derived progenitor cells in a critically ill two-yr-old child with dilated cardiomyopathy, Pediatr Transplant 13 (5) (2009) 620-623.

[4] B. Assmus, U. Fischer-Rasokat, J. Honold, F.H. Seeger, S. Fichtlscherer, T. Tonn, et al., Transcoronary transplantation of functionally competent BMCs is associated with a decrease in natriuretic peptide serum levels and improved survival of patients with chronic postinfarction heart failure: Results of the TOPCARE-CHD Registry, Circ. Res. 100 (8) (2007) 1234-1241.

[5] R. Olguntürk, S. Kula, G.T. Sucak, M.E. Ozdoğan, D. Erer, A. Saygili, Peripheric stem cell transplantation in children with dilated cardiomyopathy: Preliminary report of first two cases, Pediatr Transplant 14 (2) (2010) 257-260.

[6] J. Rivas, J.J. Menedez, R. Arrieta, J. Alves, M.P. Romero, L. Garcia-Guereta, et al., Usefulness of intracoronary therapy with progenitor cells in patients with dilated cardiomyopathy: Bridge or alternative to heart transplantation, Anales de Pediatria 74 (4) (2011) 218-225.

[7] P. Menasche, A.A. Hagege, M. Scorsin, B. Pouzet, M. Deshos, D. Duboc, Myoblast transplantation for heart failure, Lancet 357 (9252) (2001) 279-280.

[8] K. Hamano, M. Nishida, K. Hirata, A. Mikamo, M. Harada, Local implantation of autologous bone marrow cells for therapeutic angiogenesis in patients with ischemic heart disease: Clinical trial and preliminary results, Jpn. Circ. J. 65 (9) (2001) 845-847.

[9] C. Stamm, B. Westphal, H.D. Kleine, M. Petzsch, C. Kittner, Autologous bone-marrow stem cell transplantation for myocardial regeneration, Lancet 361 (9351) (2003) 45-46

[10] H.F. Tse, Y.L. Kwong, J.K. Chan, G. Lo, C.L. Ho, C.P. Lau, Angiogenesis in ischemic myocardium by intramyocardial autologous bone marrow mononuclear cell implantation, Lancet 361 (9351) (2003) 47-49.

[11] P.C. Smits, R.J. van Geuns, D. Poldermans, M. Bountioukas, Catheter-based intramyocardial injection of autologous skeletal myoblasts as a primary treatment of ischemic heart failure: Clinical experience with six-month follow-up, J. Am. Coll. Cardiol. 42 (12) (2003) 2063-2069.

[12] E.C. Perin, H.F. Dohmann, R. Borojevic, S.A. Silva, A.L. Sousa, Transendocardial, autologous bone marrow cell transplantation for severe chronic ischemic heart failure, Circulation 107 (18) (2003) 2294-2302.

[13] K. Minami, E. von Knyphausen, T. Niino, U. Blanz, G. Tenderich, S. Wlost, et al., Long-term results of pediatric heart transplantation, Ann. Thorac. Cardiovasc. Surg. 11 (6) (2005) 386-390.

[14] J.A. Towbin, Cardiomyopathy and heart transplantation in children, Curr. Opin. Cardiol. 17 (3) (2002) 274-279.

[15] J. Lindenfeld, P.A. Kelley, The Mechanical Heart in FelixB. Engeli "Heart Regeneration", World Scientific Publ. Co Pte. Ltd., Singapore, 2012, pp. 270-292.

[16] L.B. Pauliks, A. Undar, New devices for pediatric circulatory support, Curr. Opin. Cardiol. 23 (2) (2008) 91-96.

[17] R. Pfister, C.A. Schneider, ESC guidelines for the diagnosis and treatment of acute and chronic heart failure 2008: Application of natriuretic peptides, Eur. Heart. J. 30 (3) (2009) 382-383.

[18] C.G. Cowley, J.S. Lozier, G.S. Orsmond, R.E. Shaddy, Safety of endomyocardial biopsy in children, Cardiol 
Young 13 (5) (2003) 404-407.

[19] H. Kuhn, T. Lawrenz, G. Beer, Indication for myocardial biopsy in myocarditis and dilated cardiomyopathy, Med. Klin. (Munich) 100 (9) (2005) 553-561.

[20] A. Lacis, A. Erglis, Intramyocardial administration of autologous bone marrow mononuclear cells in a critically ill child with dilated cardiomyopathy, Cardiol. Young 21 (1) (2011) 110-112.

[21] A. Lacis, I. Bergmane, V. Ozolins, I. Lubaua, V. Groma, E. Ligere, et al., First results of using stem cell transplantation for pediatric patients in case of dilated cardiomyopathy, Acta. Chir. Latv. 10 (2) (2011) 76-79.

[22] Y. Zhou, S. Wang, Z. Yu, R.F.Jr. Hoyt, V. Sachdev, P. Vincent, et al., Direct injection of autologous mesenchymal stromal cells improves myocardial function, Biochem. Biophys. Res. Commun. 390 (3) (2009) 902-907.

[23] M. Mirotsou, T.M. Jayawardena, J. Schmeckpeper, M. Gnecchi, V.J. Dzau, Paracrine mechanisms of stem cell reparative and regenerative actions in the heart, J. Mol. Cell Cardiol. 50 (2) (2011) 280-289. 